\title{
Correction to: High-Yielding Radiosynthesis of [68 Ga]Ga-PSMA-11 Using a Low-Cost Microfluidic Device
}

Xin Zhang ${ }^{1}$, Fei Liu ${ }^{2,3}$, Adria C. Payne ${ }^{2,3}$, Michael L. Nickels ${ }^{2,3,4,5}$, Leon M. Bellan ${ }^{1,6}$, and H. Charles Manning ${ }^{2,3,4}$

1Department of Mechanical Engineering, Vanderbilt University, Nashville, TN 37235, USA

${ }^{2}$ Vanderbilt Center for Molecular Probes, Vanderbilt University Medical Center, Nashville, TN 37232, USA

${ }^{3}$ Vanderbilt University Institute of Imaging Science, Vanderbilt University Medical Center, Nashville, TN 37232, USA

${ }^{4}$ Department of Radiology and Radiological Sciences, Vanderbilt University Medical Center, Nashville, TN 37232, USA

${ }^{5}$ School of Medicine, Department of Radiology, Washington University in St. Louis, St. Louis, MO 63110, USA

${ }^{6}$ Departments of Biomedical Engineering, Vanderbilt University, Nashville, TN 37235, USA 2021

Correction to: Mol Imaging Biol (2020) 22:1370-1379 https://doi.org/10.1007/s11307-020-01515-7

This article was updated to correct the captions to Figures 2 and 3, which were reversed.

Publisher's Note Springer Nature remains neutral with regard to jurisdictional claims in published maps and institutional affiliations. 